\title{
ANALYSIS OF SOLDER PASTE SCOOPING WITH HIERARCHICAL POINT PROCESSES
}

\author{
Csaba Benedek
}

\author{
Distributed Events Analysis Research Group, Computer and Automation Research Institute \\ H-1111, Budapest, Kende utca 13-17, e-mail: bcsaba@sztaki.hu \\ Dept. of Electronics Technology, Budapest University of Technology and Economics \\ H-1111 Budapest, Goldmann tér 3
}

\begin{abstract}
In this paper we introduce a probabilistic approach for optical quality checking of Solder Pastes (SP) in Printed Circuit Boards (PCB). Dealing with unregistered image inputs, the task is to address at the same time SP identification, and detecting special soldering errors, called scooping. For this reason we introduce a novel Hierarchical Marked Point Process (HMPP) framework, which is able to handle the paste and scooping extraction problems simultaneously, so that the SPs and the included scoops have a parent-child relationship. A global optimization process attempts to find the optimal configuration of entities, considering the observed data, prior knowledge, and interactions between the neighboring circuit elements. The proposed method is evaluated on a real PCB image set containing more than 3000 SPs and 600 scooping artifacts
\end{abstract}

Index Terms - Marked point process, PCB, scooping

\section{INTRODUCTION}

Automatic optical inspection (AOI) is a crucial step in the manufacturing process of Printed Circuit Boards (PCBs). The quickly increasing resolution, quality and speed of the industrial cameras have recently opened several new prospects in image based verification. At finer scales a significant amount of information is revealed, which calls for shifting from simple segmentation [1] or morphology based [2] investigations towards a hierarchical modeling approach of the PCB structure, focusing jointly on circuit regions, individual Circuit Elements (CEs), CE interactions and relevant CE parts.

This paper deals with solder paste \& scooping extraction, which derives from the above multiscale problem domain. Nowadays the most widespread assembling technology of electronic circuit modules applies reflow soldering [3].

The work of the author was partially supported by the János Bolyai Research Scholarship of the Hungarian Academy of Sciences and by the Hungarian Research Fund Coherent attributes in the interpret. and percept. of the visual world (OTKA-80352). This work is connected to the scientific program of the "Development of quality-oriented and harmonized R+D+I strategy and functional model at BME" project.
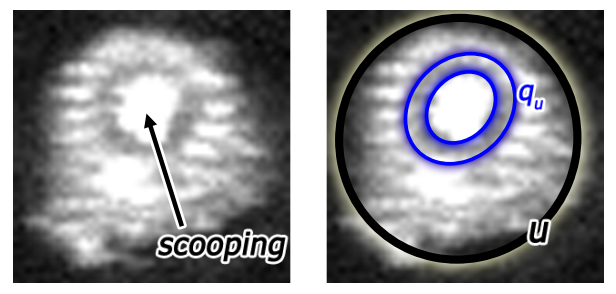

Fig. 1. Solder paste $u$ with a scoop $q_{u}(10 \mu m$ res. image)

The amount of solder paste deposited affects the reliability and strength of the reflowed solder joint [4]. Solder paste scooping (see Fig. 1) occurs when the squeegee blade enters into the stencil aperture and removes paste from the center of the print. This is caused by high print pressure and can be exaggerated by rubber blades and large stencil apertures. A single scoop in a PCB does not cause a critical quality problem, however, if the number and summarized volume of such artifacts surpass a given threshold, the board should be withdrawn. Since the PCBs are moving continuously on conveyor belt, the optical verifying system must deal with unregistered image inputs. As well, due to lens aberrations, the local contrast of the image is usually inhomogeneous, and we may observe regions that exhibit defocus blur. This effects results in varying sharpness of the scoops, therefore instead of developing global appearance models, the scoops should be rather described locally with respect to their covering solder pastes.

Several previous AOI methods use mathematical morphology [2, 5] for investigating geometric structures in binary images. Usually a series of morphological transformations is applied such as erosions, geodesic dilations, reconstruction by dilation, anchored skeletonisation, etc. The main weak point of the these techniques is that they critically rely on the input binarization step performed by thresholding the grayscale input. However, estimating appropriate global or local thresholds may be difficult, as well as due to contrast defects and slight illumination variations, the separation of CEs from the background can be imperfect [5].

In the recent years, Marked Point Processes (MPP) [6, $7,8]$ have become popular in $2 \mathrm{D}$ and $3 \mathrm{D}$ object detection, since they can efficiently model a population of an unknown 
number of entities, and consider the geometry of the objects. However, conventional MPP models do not handle object hierarchy, thus they are inappropriate to model complex multilevel scenarios. Stepping forward, we describe here the hierarchy between solder paste objects and internal scoops as a parent-child relationship embedded into a novel Hierarchical MPP (HMPP) framework. The appearance of a child object is affected by its parent entity, considering geometrical and spectral constraints, such us a parent object contains the child, or the contrast within the parent object influences the intensity characteristic of the child entity. Besides, prior knowledge is exploited in interaction modeling between different objects.

\section{PROBLEM DEFINITION}

The input of the proposed framework is an image taken from a PCB, having a 2D pixel lattice $S$. The goal is to extract and separate the Solder Pastes (SP) in the PCBs, meanwhile to detect and describe the Scoop Objects (SO) appearing in some of the pastes (Fig. 1). We model a SP by an ellipse; and a SO by two concentric ellipses as it consists of a bright central region (inside the internal ellipse) and a relatively darker elliptical ring (region between the internal and external ellipses) enclosed by the brighter SP patch (Fig. 1). In practise, noisy samples may make the recognition challenging (Fig. 5).

A given SP ellipse, denoted by $u$, is described by five geometric parameters: $x$ and $y$ center coordinates in the $S$ lattice, semimajor resp. semiminor axes, and the orientation angle. In addition, $u$ may contain a SO, $q_{u}$, which is determined by its center pixel, semi-axes of the internal and external ellipses, respectively, and the scoop orientation. We consider in the following $q_{u}$ as a child object assigned to each $u$, and denote by $q_{u}=$ nil if paste $u$ contains no scoop.

\section{HIERARCHICAL MARKED POINT PROCESS MODEL}

We model the PCB image as composition of an arbitrary number of SPs whose positions and geometric parameters are realizations of a MPP [6]. As a novelty of our approach, the scoops realize an embedded MPP within the SPs. Let us denote by $\Omega$ the space of all configurations of a finite number of SP objects. We will refer to a given object configuration in $\Omega$ by $\omega$, where $\omega=\emptyset$ or $\omega=\left\{u_{1}, \ldots, u_{n}\right\}$ for an arbitrary positive integer $n$. We also define a neighborhood relation in the SP object space: $u \sim v$ if their ellipses intersect.

Next, we introduce a $\Phi(\omega)$ energy function on the $\Omega$ configuration space, so that we aim to fulfill that the optimal configuration exhibits the minimal energy. The energy function takes into account the interactions between the geometric objects (the prior energy $\Phi_{p}(\omega)$ ), and the way they fit to the optical data (the data energy $\Phi_{d}(\omega)$ ):

$$
\Phi(\omega)=\Phi_{p}(\omega)+\Phi_{d}(\omega)
$$

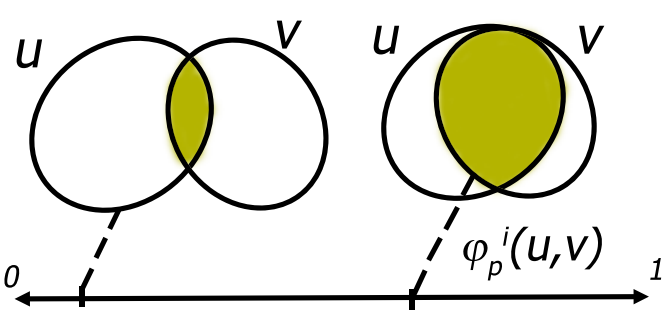

(a) Intersection area at parent level

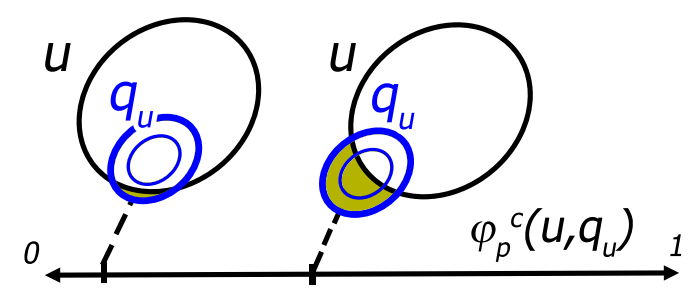

(b) Scoops overhanging area w.r.t the parent SP object

Fig. 2. Calculation of the prior terms

We begin with the $\Phi_{p}(\omega)$ energy part, which prescribes prior geometric constraints in the model. Since we aim to extract individual SP entities, we must penalize overlapping between different SP ellipses. Thus, the energy term of parent-level interactions penalizes object pairs according to the $\varphi_{p}^{i}(u, v)$ normalized overlapping area as shown in Fig. 2(a). On the other hand, we should also penalize if a scoop $q_{u}$ overhang its parent paste $u$ : this is measured by the $\varphi_{p}^{c}\left(u, q_{u}\right)$ overhanging area normalized by the area of the scoop (see Fig. 2(b)). By definition, we use $\varphi_{p}^{c}(u$, nil $)=0$. Finally, the complete prior energy term of the population is calculated as:

$$
\Phi_{p}(\omega)=\sum_{\substack{u, v \in \omega \\ u \sim v}} \varphi_{p}^{i}(u, v)+\sum_{u \in \omega} \varphi_{p}^{c}\left(u, q_{u}\right)
$$

The data energy associated with object $u$ is decomposed into a parent term $\varphi_{d}^{p}(u)$ and a child term $\varphi_{d}^{c}\left(u, q_{u}\right)$. In the PCB images the parent SPs can be modeled as bright ellipses surrounded by darker background. To evaluate the contrast between the pastes and the board, we calculate the Bhattacharya [6] distance $d_{B}(u)$ between the pixel intensity distributions of the internal SP regions and their boundaries:

$$
d_{B}(u)=1-\int \sqrt{\lambda_{u}^{\text {in }}(x) \cdot \lambda_{u}^{\text {out }}(x)} d x
$$

where $\lambda_{u}^{\text {in }}(x)$ (resp. $\lambda_{u}^{\text {out }}(x)$ ) is the empirical gray level distribution of the pixels belonging to $u$ (resp. a concentric elliptical ring around $u$ ), as shown in Fig. 3. The data energy $\varphi_{d}^{p}(u)$ associated with the parent object $u$ is then given by: $\varphi_{d}^{p}(u)=Q\left(d_{B}(u), d_{0}\right)=$

$$
= \begin{cases}\left(1-\frac{d_{B}(u)}{d_{0}}\right) & \text { if } d_{B}(u)<d_{0} \\ \exp \left(-\frac{d_{B}(u)-d_{0}}{0.1}\right)-1 & \text { if } d_{B}(u) \geq d_{0}\end{cases}
$$




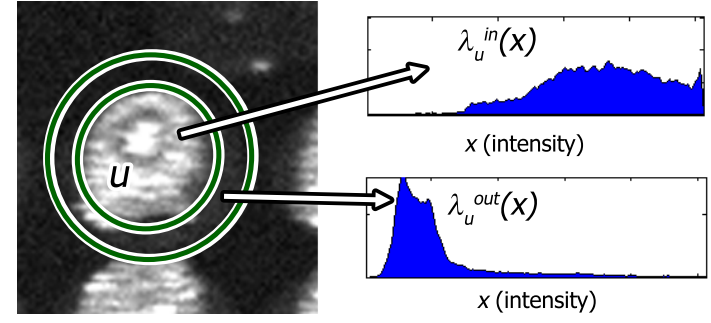

Fig. 3. Utility of the $\lambda_{u}^{\text {in }}(x)$ and $\lambda_{u}^{\text {in }}(x)$ histograms for the parent data term calculation

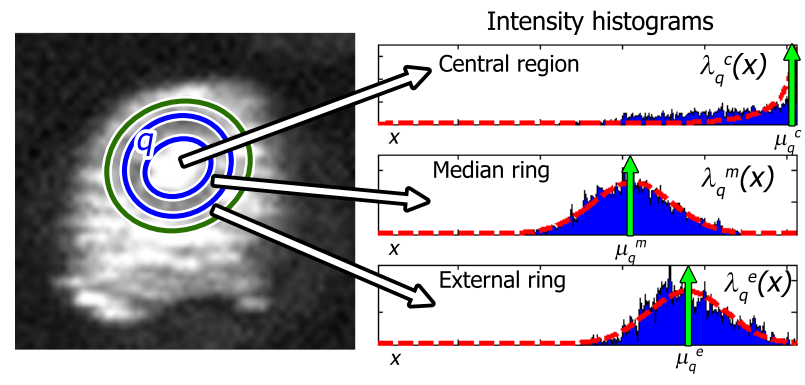

Fig. 4. Child data term calculation

where $d_{0}$ is the object-acceptance threshold set by a Maximum Likelihood (ML) parameter estimation process based on training regions [7].

The construction of the child's data term $\varphi_{d}^{c}\left(u, q_{u}\right)$ is based on similar principles. We use $\varphi_{d}^{c}(u$, nil $)=0$, otherwise we distinguish three regions of each scoop: the central bright ellipse, the darker median ring and the bright external ring, as shown in Fig. 4. Experimental evidences prove, that for a real scoop $q$, the gray level histogram of the central region, $\lambda_{q}^{\mathrm{c}}(x)$ follows a skewed distribution, while the medium and external region histograms $\left(\lambda_{q}^{\mathrm{m}}(x)\right.$ resp. $\left.\lambda_{q}^{\mathrm{e}}(x)\right)$ can be approximated by Gaussian densities. Let us denote by $\mu_{q}^{\mathrm{c}}, \mu_{q}^{\mathrm{m}}$ resp. $\mu_{q}^{\mathrm{e}}$ the peak locations of the smoothed $\lambda_{q}^{\mathrm{c}}(x), \lambda_{q}^{\mathrm{m}}(x)$ resp. $\lambda_{q}^{\mathrm{e}}(x)$ functions. We prescribe three constraints for an efficient scoop candidate: (i) it exhibits high $\mu_{q}^{\mathrm{c}}$ value, and intensity ratios (ii) $\mu_{q_{u}}^{\mathrm{c}} / \mu_{q_{u}}^{\mathrm{m}}$ resp. (iii) $\mu_{q_{u}}^{\mathrm{e}} / \mu_{q_{u}}^{\mathrm{m}}$ pass given contrast thresholds $d^{\mathrm{cm}}$ and $d^{\mathrm{em}}$, which are set by ML training. To enforce the simultaneous fulfilment of the (i)-(iii) properties, the child's data-energy value is calculated using the maximum operator (logical AND) from the subterms of the three constraints:

$$
\begin{aligned}
\varphi_{d}^{c}\left(u, q_{u}\right)=\max ( & Q\left(\mu_{q_{u}}^{\mathrm{c}}, d^{c}\right), \\
& Q\left(\mu_{q_{u}}^{\mathrm{c}} / \mu_{q_{u}}^{\mathrm{m}}, d^{\mathrm{cm}}\right), \\
& \left.Q\left(\mu_{q_{u}}^{\mathrm{e}} / \mu_{q_{u}}^{\mathrm{m}}, d^{\mathrm{em}}\right)\right)
\end{aligned}
$$

Finally, the data energy of the configuration is obtained as

$$
\Phi_{d}(\omega)=\sum_{u \in \omega} \varphi_{d}^{p}(u)+\varphi_{d}^{c}\left(u, q_{u}\right)
$$

\section{OPTIMIZATION}

For the estimation of the optimal object configuration $\hat{\omega}=$ $\operatorname{argmin}_{\omega \in \Omega}\left(\Phi_{p}(\omega)+\Phi_{d}(\omega)\right)$ we have developed a hierarchical extension of the recent Multiple Birth and Death Algorithm [6]. The steps are as follows:

Initialization: start with an empty population $\omega=\emptyset$.

Main program: initialize the inverse temperature parameter $\beta=\beta_{0}$ and the discretization step $\delta=\delta_{0}$ and a constant basic birth frequency $b_{0}$. Alternate the following four steps, till convergence is reached:

1) Parent Birth step: Visit all pixels of the $S$ lattice one after the other. At each pixel $s \in S$ call $S P$ Generation procedure with probability $\delta \cdot b_{0}$, so that create a new SP object $u$ with center $s$; set $u$ 's axis length and orientation parameters randomly following prior size distributions; use null-scoop initially: $q_{u}=$ nil and add $u$ to the current configuration $\omega$.

2) Parent Death step: Consider the current configuration $\omega$ and create a list of the $u \in \omega$ objects sorted from the highest to the lowest $\varphi_{d}^{p}(u)$ values. For each object $u$ taken in this order, compute the cost of deleting $u$ from $\omega$ w.r.t. the global configuration energy:

$$
\begin{aligned}
\Delta \Phi_{\omega}^{u} & =\Phi(\omega /\{u\})-\Phi(\omega)= \\
& =-\varphi_{d}^{p}(u)-\varphi_{d}^{c}\left(u, q_{u}\right)-\varphi_{p}^{c}\left(u, q_{u}\right)-\sum_{\substack{v \in \omega \\
v \sim u}} \varphi_{p}^{i}(u, v)
\end{aligned}
$$

Next, we derive the $p_{d}(u)$ death rate as follows:

$$
p_{d}(u)=f\left(\Delta \Phi_{\omega}^{u}\right)=\frac{\delta \cdot \exp \left(-\beta \cdot \Delta \Phi_{\omega}^{u}\right)}{1+\delta \cdot \exp \left(-\beta \cdot \Delta \Phi_{\omega}^{u}\right)}
$$

Finally, we remove $u$ from $\omega$ with a probability $p_{d}(u)$.

3) Child Maintenance step: for each $u$ object in $\omega$, we generate a new scoop candidate $q_{u}^{\prime}$ which is either $q_{u}^{\prime}=$ nil; or we pick up a random point covered by the ellipse of $u$, and appoint it as the center of $q_{u}^{\prime}$, while orientation and axes parameters of $q_{u}^{\prime}$ are set randomly. Thereafter, we calculate the energy cost of exchanging $q_{u}$ to $q_{u}^{\prime}$ :

$$
\Delta \varphi\left(u, q_{u}, q_{u}^{\prime}\right)=\varphi_{p}^{c}\left(u, q_{u}^{\prime}\right)+\varphi_{d}^{c}\left(u, q_{u}^{\prime}\right)-\left(\varphi_{d}^{c}\left(u, q_{u}\right)+\varphi_{p}^{c}\left(u, q_{u}\right)\right)
$$

The scoop exchange likelihood is calculated using the $f($. function defined by (2): $p_{c}(u)=f\left(\Delta \varphi\left(u, q_{u}, q_{u}^{\prime}\right)\right)$. Then with a probability $p_{c}(u)$, we replace $q_{u}$ with $q_{u}^{\prime}$.

4) Convergence test: if the process has not converged yet, increase the inverse temperature $\beta$ and decrease the discretization step $\delta$ with a geometric scheme, and go back to the Parent Birth step. The convergence is obtained when all the SP objects added during the birth step, and only these ones, have been killed during the death step, meanwhile the Child Maintenance does not report any more changes. 


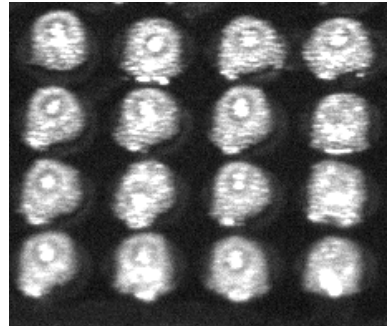

(a)

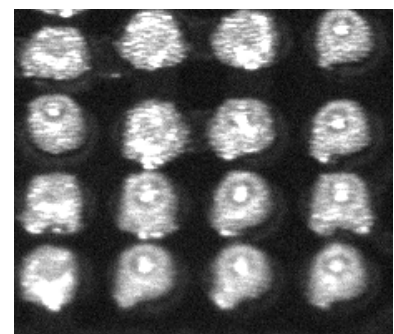

(c)

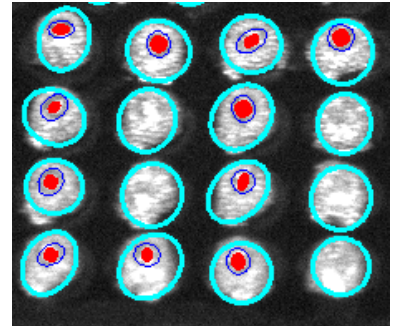

(b)

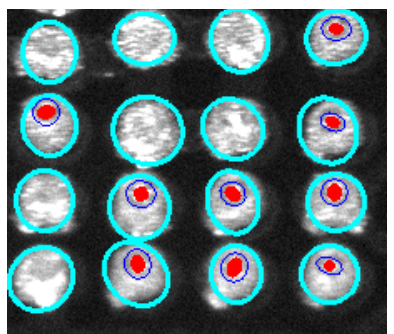

(d)
Fig. 5. Two image parts and the results

Table 1. Quantitative evaluation results of the joint solder paste \& scoop extraction with the proposed model. The used PCB test set contains 3040 SP entities and 613 SOs.

\begin{tabular}{|l||l|l||l|l|l|}
\hline \multicolumn{1}{|c||}{} & \multicolumn{2}{c|}{ SP level } & \multicolumn{3}{c|}{ SO level } \\
\hline Method & Rc & Pc & Rc & Pc & FM \\
\hline \hline Morph & $98.6 \%$ & $100 \%$ & $78.8 \%$ & $65.2 \%$ & $71.4 \%$ \\
\hline HMPP & $99.5 \%$ & $99.9 \%$ & $90.7 \%$ & $91.6 \%$ & $91.1 \%$ \\
\hline
\end{tabular}

\section{EVALUATION}

We have tested our method on a real PCB image set which consists of $10 \mu \mathrm{m}$ resolution photos of 40 different circuits, each of them contains 76 SPs. In aggregate 613 SOs are present in the $3040(=40 \cdot 76)$ solder pastes of the test set. The quality of the different PCBs shows a large variety: 18 out of the 40 analysed images contain less than 10 scoops/board, 14 circuits include 10-30 scoops, and each of the remaining 8 ones holds more than 30 SOs. Meanwhile, the test images exhibit significantly different and spatially varying contrast due to the quick motion of the production line and the optical limitations of the industrial cameras.

Some qualitative results of the hierarchical SP-SO extraction are shown in Fig. 5. We have fulfilled the quantitative evaluation at entity level: we have calculated the Recall (Rc) and Precision (Pc.) rates of the SP and SO detection respectively, results can be observed in Table 1. Minor errors of SP detection are caused by notably blurred image regions and noise patches in some of the images. The challenging part of the process proved to be the scoop identification, however, the detection results are here above $90 \%$ as well. We also provide the F-measure (FM in Table 1) [7] of the detection, which is the harmonic mean of precision and recall. In Fig. 5, we can observe in each image one false negative scoop, which is resulted by the strongly overlapping gray level histograms of the median and external rings. This is currently a limitation of the method.

As a reference, we have implemented a morphology based approach from similar processing steps to [2], and compared the performance in the test image set. Results in Table 1 show the superiority of the proposed model over the morphology based technique. Circuit technologists have confirmed, that based on our reported accuracy a fair statistical analysis of the scooping effects can be performed for quality characterization of the PCBs.

\section{CONCLUSION}

We have proposed a Hierarchical Marked Point Process (HMPP) framework for optical scooping analysis in circuit boards. The method incorporates entitiy detection and quality validation in a joint probabilistic approach. The author would like to thank László Jakab and Olivér Krammer for provision of the input images and for their kind remarks and advice regarding the technical aspects of the problem and task definition.

\section{REFERENCES}

[1] D. Comaniciu and P. Meer, "Mean shift: A robust approach toward feature space analysis," IEEE Trans. on Pattern Anal. and Machine Intelligence, vol. 24, pp. 603-619, 2002.

[2] P. Soille and P. Vogt, "Morphological segmentation of binary patterns," Pattern Recogn. Lett., vol. 30, no. 4, pp. 456-459, 2009.

[3] O. Krammer and B. Sinkovics, "Improved method for determining the shear strength of chip component solder joints," Microelectronics Reliability, vol. 50, no. 2, pp. 235 - 241, 2010.

[4] S.H. Mannan, N.N. Ekere, E.K. Lo, and I. Ismail, "Predicting scooping and skipping in solder paste printing for reflow soldering of smt devices," Soldering \& Surface Mount Technology, vol. 5, no. 3, pp. $14-17,1993$.

[5] Z. Li, J. Yang, C. Liu, G. Liu, and Y. Cheng, "Unsupervised range-constrained thresholding," Pattern Recogn. Lett., vol. 32, no. 2, pp. 392-402, 2011.

[6] X. Descombes, R. Minlos, and E. Zhizhina, "Object extraction using a stochastic birth-and-death dynamics in continuum," $J$. Math. Imaging and Vision, vol. 33, pp. 347-359, 2009.

[7] C. Benedek, X. Descombes, and J. Zerubia, "Building extraction and change detection in multitemporal remotely sensed images with multiple birth and death dynamics," in IEEE WACV, Snowbird, USA, 2009.

[8] S. Descamps, X. Descombes, A. Béchet, and J. Zerubia, “Automatic flamingo detection using a multiple birth and death process," in Proc. IEEE ICASSP, 2008. 